\title{
Guiding light via geometric phases
}

\author{
Sergei Slussarenko, ${ }^{1,2}$ Alessandro Alberucci, ${ }^{3,4}$ Chandroth P. Jisha, ${ }^{5}$ Bruno \\ Piccirillo, ${ }^{1}$ Enrico Santamato, ${ }^{1}$ Gaetano Assanto, ${ }^{3,4}$ and Lorenzo Marrucci ${ }^{1}$ \\ ${ }^{1}$ Dipartimento di Fisica, Università di Napoli Federico II, Napoli, Italy \\ ${ }^{2}$ Centre for Quantum Dynamics and Centre for Quantum Computation and Communication Technology, \\ Griffith University, Brisbane, Queensland 4111, Australia \\ ${ }^{3}$ Nonlinear Optics and OptoElectronics Lab, University Roma Tre, I-00146 Rome, Italy \\ ${ }^{4}$ Optics Lab, Department of Physics, Tampere University of Technology, FI-33101 Tampere, Finland \\ ${ }^{5}$ Centro de Física do Porto, Faculdade de Ciências, \\ Universidade do Porto, 4169-007 Porto, Portugal
}

(Dated: December 4, 2015)

\begin{abstract}
Known methods for transverse confinement and guidance of light can be grouped into a few basic mechanisms, the most common being metallic reflection, total internal reflection and photonicbandgap (or Bragg) reflection 1 [15. All of them essentially rely on changes of the refractive index, that is on scalar properties of light. Recently, processes based on "geometric Berry phases", such as manipulation of polarization states or deflection of spinning-light rays ${ }^{6}$, have attracted considerable interest in the contexts of singular optics and structured light $10[11$. Here, we disclose a new approach to light waveguiding, using geometric Berry phases and exploiting polarization states and their handling. This can be realized in structured three-dimensional anisotropic media, in which the optic axis lies orthogonal to the propagation direction and is modulated along it and across the transverse plane, so that the refractive index remains constant but a phase distortion can be imposed on a beam. In addition to a complete theoretical analysis with numerical simulations, we present a proof-of-principle experimental demonstration of this effect in a discrete element implementation of a geometric phase waveguide. The mechanism we introduce shows that spin-orbit optical interactions can play an important role in integrated optics and paves the way to an entire new class of photonic systems that exploit the vectorial nature of light.
\end{abstract}

Waveguides are central to modern photonics and optical communications. Besides the standard optical fibers -based on total internal reflection (TIR) and gradedindex (GRIN) refractive potential- and the hollow-metalpipes for microwaves 1 , several more complex structures have been investigated, ranging from photonicbandgap systems ${ }^{2}[5]$ to "slot" waveguides ${ }^{12}$, plasmonic waveguides 13 , coupled-resonators $14 \mid 15$, gratingmediated $\frac{16}{16}$ and Kapitza-effect waveguides $\frac{17}{17}$ Despite such variety, all light-guiding mechanisms investigated hitherto rely on variations, sudden or gradual, of the refractive index or -generally- the dielectric permittivity. Even when anisotropic materials are employed to realize waveguides, as for example in liquid crystals 18 , light confinement is based on the transverse modulation of the refractive index experienced by extraordinary waves through the nonuniform orientation of the optic axis with respect to the wave-vector. A fundamental question is whether the guided propagation of light can be achieved at all in structures without perturbations of the refractive index. As we shall prove, this is indeed possible provided that the transverse trapping is purely based on vectorial effects, that is, it relies on spin-orbit interactions between wave propagation and polarization states of light 19 : otherwise stated, an entirely new mechanism for light confinement.

Spin-orbit photonic interactions are strictly related to geometric Berry phases. In the context of optics, the latter are phase retardations linked exclusively to the geometry of the transformations imposed to light by the medium and independent of the optical path length 19 . This concept has been already implemented in optical elements with various architectures, including patterned dielectric gratings, liquid crystals and metasurfaces 20 24. These devices exploit the medium anisotropy to modulate the polarization state of light in a space-varying manner across the plane transverse to propagation. This, in turn, gives rise to a spatially inhomogeneous Pancharatnam-Berry (PB) phase ${ }^{8 / 25}$, resulting in a reshaped optical wavefront. Hence, a PB optical element (PBOE) behaves as a phase mask, despite exhibiting a constant refractive index and a transverselyuniform optical path length, that is a flat geometry.

In this work we disclose the possibility of guiding electromagnetic waves (and in particular light) by exploiting $\mathrm{PB}$ phases in a bulk, that is an extended continuous medium supporting beam propagation over several Rayleigh distances. As we will show, at variance with PBOEs this requires birefringent materials whose optic axis is modulated both in the transverse plane and in the longitudinal coordinate along the propagation direction, a medium structured in three-dimensions (3D).

We investigate light propagation along the axis $z$ of an inhomogeneous uniaxial dielectric. The optic axis $\hat{u}$ is assumed to be space-varying, but lying everywhere in the $x y$ plane transverse to propagation. Its point-wise orientation is described by the angle $\theta(x, y, z)$ between $\hat{u}$ and the $y$ axis in the laboratory frame (see Fig. 1a). We also assume that the principal values $\epsilon_{\|}$and $\epsilon_{\perp}$ of the (relative) permittivity tensor are uniform, corresponding 

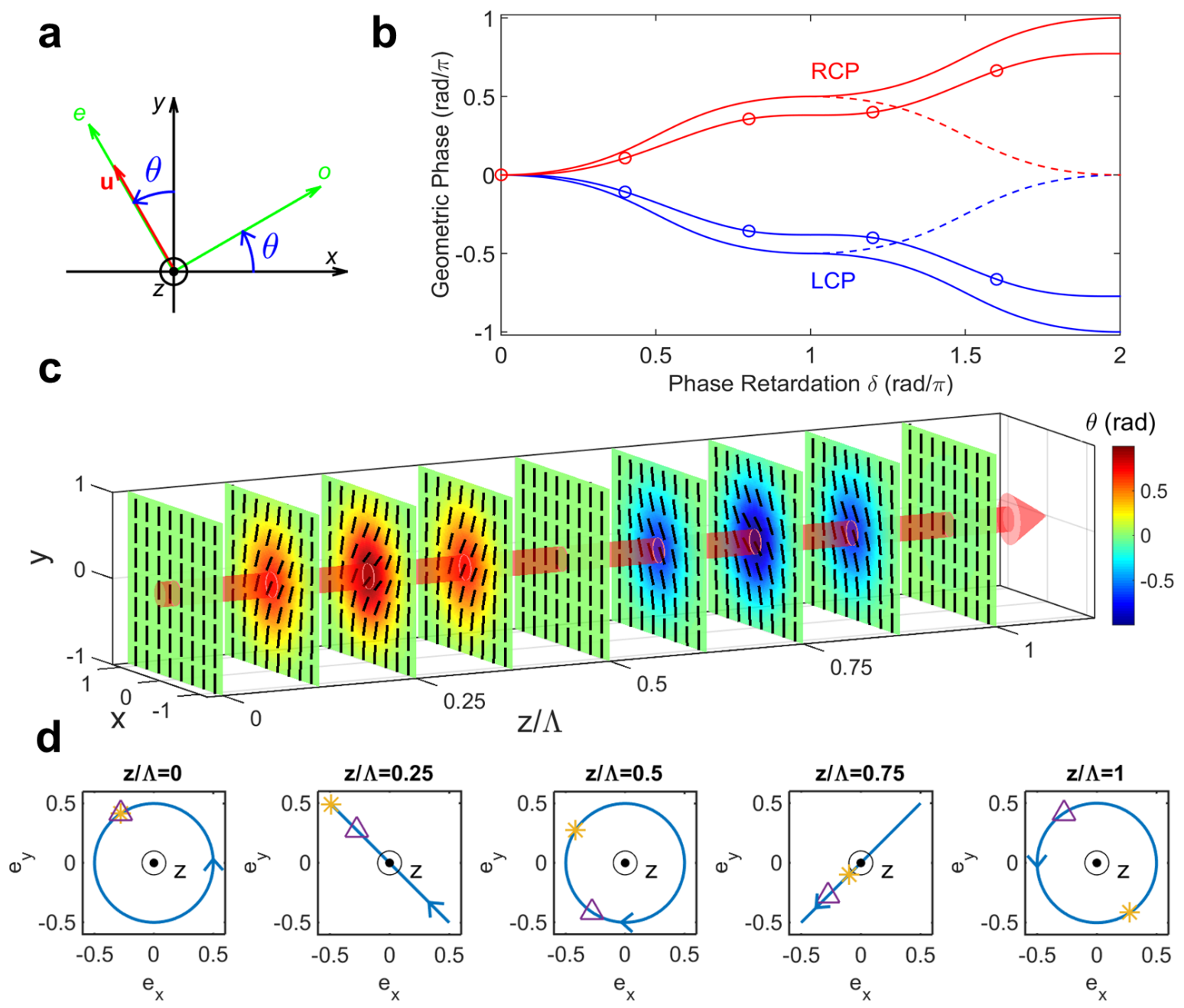

FIG. 1. Geometric-phase waveguide concept. a, Reference system with orientation of the optic axis $\boldsymbol{u}$ and ordinary/extraordinary $(o / e)$ field directions in the laboratory frame $x y z$; the angle $\theta$ between $\boldsymbol{u}$ and the axis $y$ varies from point to point. $\mathbf{b}$, Geometric phase acquired by a plane wave, $\mathrm{CP}$ at the input, propagating along $z$ in a transversely homogeneous medium with $\theta=\pi / 4$ as a function of the birefringence retardation $\delta(z)$, relative to the case with $\theta=0$. The geometric phase sign is fixed by the CP input handedness (blue and red lines). If $\theta$ is uniform along $z$ (dashed lines), the gometric phase reaches a maximum (in the example $\pi / 2$ ) when $\delta=\pi$ and then decreases to zero for $\delta=2 \pi$. If the angle $\theta$ is suddenly inverted at $\delta=\pi$ (solid lines), the phase grows monotonically. If $\theta$ is sinusoidally modulated along $z$ (solid lines with circles), the phase increases monotonically at a slightly lower rate than in the previous case. When launching a light beam along $z$ in a periodic uniaxial medium with $|\theta|$ larger on axis than in the outer regions, the cumulative geometric phase leads to a guiding effect for an input LCP or RCP, depending on the initial $\theta$ modulation sign. c, 3D illustration of a continuously modulated geometric-phase waveguide: the orientation of the optic axis is longitudinally sinusoidal and transversely Gaussian. We sketch nine sections within a modulation period, with the black rods representing the optic axis distribution and the colors corresponding to $\theta$; the guided light beam is represented as a red arrow. $\mathbf{d}$, A circular plane wave input beam propagates along the structure with varying polarizations which alternate between LCP and RCP, with the same periodicity of the medium $\left(e_{x}\right.$ and $e_{y}$ are normalized electric fields in the time domain). Star and triangle show the geometric phase evolution of two points in the transverse plane $\left(x_{1}, y_{1}, z\right)$ and $\left(x_{2}, y_{2}, z\right)$ located so that $\theta_{2}-\theta_{1}=\pi / 4$. After one period the overall phase difference is $\pi$, consistently with the graph in $\mathbf{b}$ (solid lines).

to constant refractive indices $n_{o}=\sqrt{\epsilon_{\perp}}$ and $n_{e}=\sqrt{\epsilon_{\|}}$ for ordinary and extraordinary eigenwaves, respectively.

Let us first recall that, in a homogeneous uniaxial with plane waves propagating with wave-vector along $z$, the ordinary and extraordinary eigenfields have amplitudes $\psi_{o}(z)=e^{i k_{0} n_{o} z} \psi_{o}(0)$ and $\psi_{e}(z)=e^{i k_{0} n_{e} z} \psi_{e}(0)$, respec- tively, with $k_{0}=2 \pi / \lambda$ the vacuum wavenumber and $\lambda$ the wavelength. In other words, the two waves propagate independently of each other and acquire a relative phase retardation $\delta(z)=k_{0} \Delta n z$ versus propagation, where $\Delta n=n_{e}-n_{o}$ is the birefringence. If we now turn from the usual ordinary/extraordinary linear polar- 
ization basis to the left/right (L/R) circular polarization (CP) basis, the same evolution for LCP/RCP wave amplitudes is described by (see Methods for a derivation):

$\psi_{L}(z)=e^{i \bar{n} k_{0} z}\left[\cos \left(\frac{\delta}{2}\right) \psi_{L}(0)-i \sin \left(\frac{\delta}{2}\right) e^{i 2 \theta} \psi_{R}(0)\right]$

$\psi_{R}(z)=e^{i \bar{n} k_{0} z}\left[\cos \left(\frac{\delta}{2}\right) \psi_{R}(0)-i \sin \left(\frac{\delta}{2}\right) e^{-i 2 \theta} \psi_{L}(0)\right]$

where $\bar{n}=\left(n_{o}+n_{e}\right) / 2$ is the average refractive index. Equations (1) point out that the two forwardpropagating circular waves evolve with a common phase $\bar{n} k_{0} z$ and, in addition, periodically exchange handedness (that is LCP becomes RCP and vice versa) acquiring an additional phase factor $\pm 2 \theta(+/-$ for initial RCP/LCP, respectively). This extra phase clearly has a geometric nature and is an example of $\mathrm{PB}$ phase $\frac{8|9| 25}{\text {. The two }}$ $\mathrm{CP}$ waves completely interchange after a propagation distance $z_{\mathrm{coh}} / 2$ such that $\delta\left(z_{\mathrm{coh}} / 2\right)=\pi$, then the process reverts and the optical field retrieves its initial state in $z=z_{\text {coh }}$ where $\delta\left(z_{\text {coh }}\right)=2 \pi$ (see Fig. 1 $1 \mathbf{b}$ ). Hence, the geometric phase in such uniform medium oscillates but does not accumulate over distance. While in PBOEs the propagation can be halted when $\delta(z)=\pi$ by properly arranging the medium length (or its birefringence), so as to obtain a non vanishing $\mathrm{PB}$ phase at the output, in a system with extended propagation length (e.g. a waveguide) the described geometric phase appears to play no significant role.

A simple equalization approach to build-up a geometric phase along $z$ consists of introducing a periodic modulation in order to counteract its recurring cancellation, analogous to dispersion-compensation in fibers and quasiphase-matching in nonlinear optics $26 \mid 27$. This requires to periodically invert the sign of $\theta$ along $z$ with the same spatial period as the "natural" interchange described above, so that the PB phase will keep adding up (with the same positive or negative sign depending on the input polarization) and accumulate monotonically over distance (Fig. 1 b). That is, we must have $\theta(z)=\theta(z+\Lambda)$ where $\Lambda=z_{\mathrm{coh}}=2 \pi /\left(\Delta n k_{0}\right)=\lambda / \Delta n$ (the average value of $\theta(z)$, even if nonzero, plays no role). The resulting $\mathrm{PB}$ phase can then be exploited to control light over an extended propagation length. In particular, to achieve light confinement the phase retardation needs to be larger on the beam axis than in the outer regions, as in TIR or GRIN optical fibers. Such a phase modulation across the beam gives rise to a focusing effect able to counteract the natural diffraction and leading to transverse confinement and guidance. By combining longitudinal $(z)$ and transverse ( $x y$ plane) modulations of $\theta$, an overall $3 \mathrm{D}$ structure described by $\theta(x, y, z)=\sigma(z) \Gamma(x, y)$ is obtained, with $\sigma(z)=\sigma(z+\Lambda)$ a periodic function to yield a monotonic growth of the geometric phase and $\Gamma(x, y)$ a transverse profile which defines the waveguide crosssection. A sample sketch of such a structure is in Fig. 11c. We stress that in this inhomogeneous anisotropic medium the optic axis $\hat{u}$ remains always orthogonal to the propagation direction, so no refractive index changes contribute to guiding.

We developed a full analytic theory of the afore described PB guiding mechanism in the frame of the slowlyvarying envelope approximation (see Methods). The main results, in a simpler geometry with one transverse coordinate $x$ for the sake of simplicity, can be summarized in a dynamical equation for the wave amplitude $A$ corresponding to a given $\mathrm{CP}$ input:

$$
i \frac{\partial A}{\partial z}=-\frac{1}{2 \bar{n} k_{0}} \frac{\partial^{2} A}{\partial x^{2}}+V(x) A
$$

where $V(x)= \pm(\pi / \Lambda) \Gamma(x)$ with $+/-$ for input $\mathrm{LCP} / \mathrm{RCP}$, respectively. Quadratic terms in $\Gamma$ have been omitted for simplicity (see Methods for the complete expression). Equation (2) is fully equivalent to a 1D Schrödinger equation for a particle oscillating in a potential $V(x)$ (with $z$ playing the role of time) or to the standard (paraxial) Helmholtz equation for light propagating in a GRIN medium with refractive index $n(x)$ such that $V(x)=-k_{0}\left[n^{2}(x)-\bar{n}^{2}\right] /(2 \bar{n})$. Depending on the sign of $\Gamma(x)$, either LCP or RCP perceive a trapping potential and get confined, while the orthogonal $\mathrm{CP}$ undergoes defocusing and diffract even faster than normal, confirming once again the spin-orbit nature of this interaction. For the focused CP component the structure behaves as a standard GRIN waveguide with index profile $n(x)$ (the $\mathrm{CP}$ state refers to the input, as the circular polarization continuously evolves between left and right during propagation, see Fig. 1dd). Examples of the calculated effective potential and corresponding guided modes are given in Fig. 2a-c. In order to check the validity of our theory we carried out finite-difference time-domain (FDTD) numerical simulations of light propagation in the waveguide structure, solving the full Maxwell equations in the space-variant birefringent medium. Figure $2 \mathrm{~d}-\mathrm{f}$ provides examples of the obtained results, in excellent agreement with the theoretical predictions.

We demonstrated this novel approach to guiding light with a proof-of-principle experiment. Rather than using the continuous structure described above, we realized a simpler "discrete-element" PB-waveguide consisting of equally spaced PBOEs alternating with a homogeneous isotropic dielectric (air); each PBOE is essentially equivalent to a thin slice of the PB-waveguide and acts as a focusing element, that is, a geometric-phase lens $(\mathrm{GPL}){ }^{9 \mid 28}$ 30. In other words, we mimicked the operation of the PB-waveguide with a sequence of equally spaced converging lenses, exclusively using PB phases (Fig. 33).

Our GPLs were thin films of a birefringent uniaxial, nematic liquid crystals, having a transverse distribution of the optic axis given by $\theta=\alpha\left(x^{2}+y^{2}\right)$, with $\alpha$ a constant, see Fig. 3b. Neglecting diffraction within the finite GPL thickness, the action of each lens on a CP input beam is described by Eqs. (1). For $\delta=\pi$, the polarization handedness is inverted at the output and 

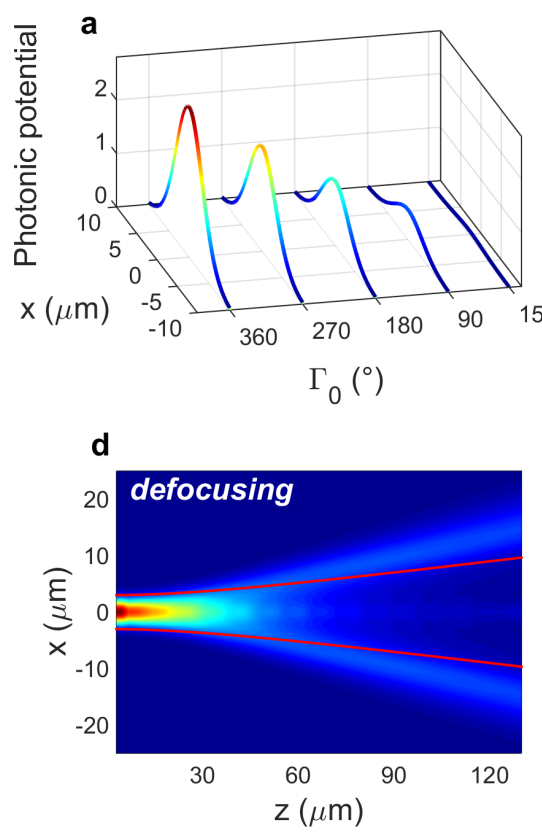
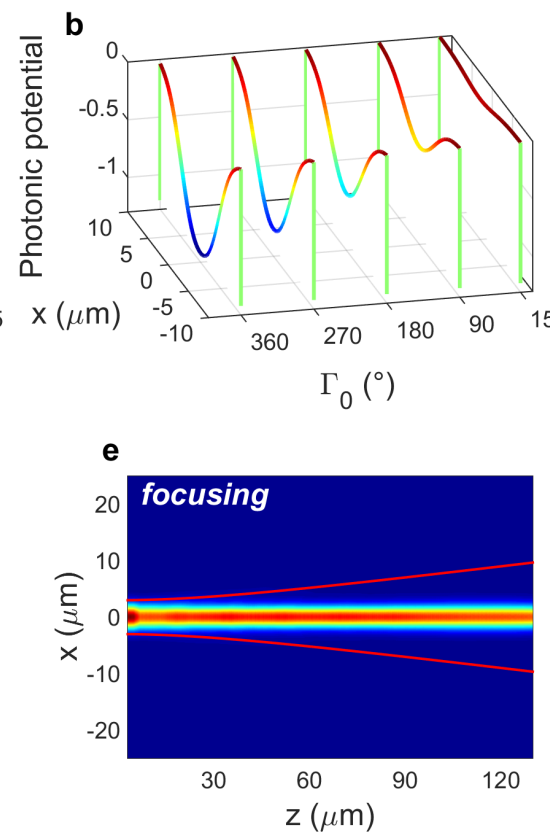
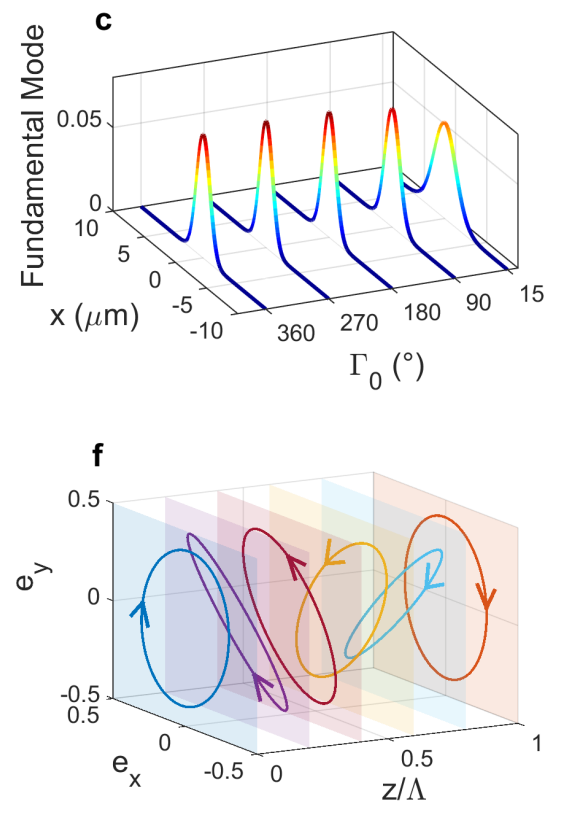

FIG. 2. Theory and simulations. a-b, Effective photonic potential $V(x) 2 \bar{n} / k_{0}$ versus $x$ and maximum $\theta$ angle $\Gamma_{0}$ (we assumed a Gaussian distribution for the transverse orientation by setting $\left.\Gamma=\Gamma_{0} \exp \left(-x^{2} / w_{D}^{2}\right)\right)$ perceived by the defocused (a, LCP input) and the confined (b, RCP input) waves, respectively. The terms proportional to $\Gamma^{2}$ are accounted for (see Methods). c, Corresponding fundamental guided mode; represented is the field amplitude versus $x$ and $\Gamma_{0}$. d-f, FDTD simulations for $\Gamma_{0}=15^{\circ}$ when the input beam is LCP $(\mathbf{d})$ and RCP $(\mathbf{e})$, respectively; the color scale gives the local light intensity; the red lines give the beam radius evolution for a homogeneous medium, that is for ordinary diffraction. $\mathbf{f}$, Evolution of the confined beam polarization state within a modulation period. Here $\lambda=1 \mu \mathrm{m}, n_{o}=1.5, n_{e}=1.7, \sigma(z)$ is sinusoidal and the transverse distribution has $w_{D}=5 \mu \mathrm{m}$.

the outgoing wave acquires a geometric phase $\pm 2 \theta(x, y)$, which is equivalent to the phase of a thin lens having focal length $f= \pm \frac{\pi}{2 \alpha \lambda}$. Hence, the GPL acts as a focusing element for one CP handedness but defocusing for the opposite one. Since the circular polarization handedness is inverted at each GPL, in order to balance out diffraction throughout the structure, we flipped the sign of $\alpha$ at each step, resulting in a longitudinal $\theta$ oscillation as for the continuous PB waveguide case. The fundamental mode of our discrete waveguide, the shape-preserving Gaussian beam that propagates with period equal to the distance $d$ between opposite lenses, has a beam waist $w_{0}=\sqrt{\lambda[(4 f-d) d]^{1 / 2} /\left(2 n_{i} \pi\right)}$ centered between subsequent GPLs, where $n_{i}$ is the refractive index of the isotropic medium between elements.

For our experiments, we set up a sequence of five GPLs, rotating every other one by $\pi$ around the $y$ axis so as to produce alternating signs of $\alpha$; moreover, exploiting the electro-optic response of nematic liquid crystals, the lenses were electrically tuned to $\delta=\pi$ (see Methods for details). We characterized light propagation in the structure for both LCP and RCP inputs and compared it with free-space propagation. As can be seen in Figs. 3k-e, the experimental results are in very good agreement with the polarization-dependent waveguiding predicted for the continuous case: only one input CP handedness was con- fined in the PB-waveguide with a shape-preserving mode, whereas the opposite polarization was radiated off almost immediately. The acquired data matched well the theory, as shown in Fig. 3:

In conclusion, we have shown, both theoretically and experimentally, that geometric Pancharatnam-Berry phases can be used for transverse confinement of electromagnetic waves, thus introducing an entirely new light-guiding principle that exploits spin-orbit optical interactions and the vectorial nature of electromagnetic radiation. Besides its fundamental interest, the proposed approach is technologically relevant for future integrated optics systems, including those involving metasurfaces 23244 . The development of novel generations of PB guided-wave photonics and manipulation of light is envisioned in dielectrics and metamaterials for the whole ultraviolet to terahertz spectrum. 

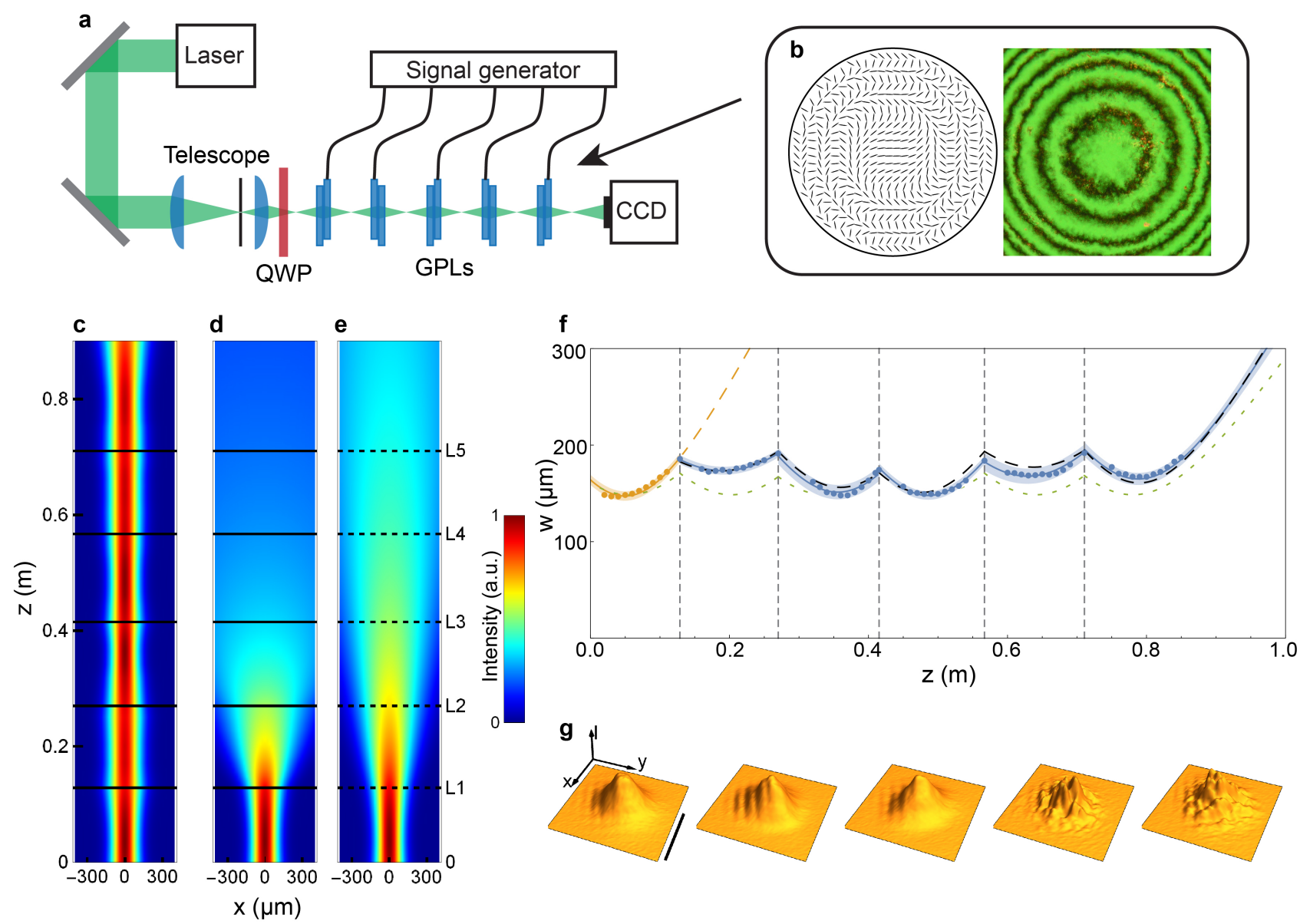

FIG. 3. Experiment. a, Experimental setup: five equally-spaced electrically-tuned GPLs form a discrete-element geometricphase waveguide. A $532 \mathrm{~nm}$ continuous-wave Gaussian beam was adjusted in transverse size with a telescope so as to match the fundamental mode of the waveguide, circularly polarized with a quarter-wave plate (QWP) and then launched into the waveguide. Beam profiles at various intermediate positions $z$ along the propagation and at the output were acquired by a movable CCD camera and used to reconstruct the mode parameters of the beam after each GPL. b, Distribution of the optic axis and corresponding microscope image of a GPL between crossed polarizers; dark fringes correspond to regions where the optic axis is aligned parallel to one of the polarizers. c-e, Data-reconstructed evolution of the propagating beam for each of the following cases: c, guided mode obtained for RCP input; d, divergent beam obtained for LCP input; e, free-space diffracting beam for the same input parameters. The color scale gives the local light intensity. Horizontal lines and L1-L5 labels indicate the GPL positions within the discrete sequence (dashed lines mark removed GPLs). f, Beam radius versus $z$ in the guided case. Dots are the measurement data, the blue solid lines are the Gaussian-beam fits for those dots between two subsequent GPLs, the blue shaded areas being the fit confidence regions at one standard deviation; the black dashed line is the theoretical prediction based on the ABCD method, also accounting for the imperfections of the Gaussian beam (as defined by the $M^{2}$ parameter) and of the GPLs; the dashed green line corresponds to the predicted beam evolution for an ideal Gaussian input, with $M^{2}=1$. Vertical dashed lines give the positions of the GPLs in the waveguide. g, Acquired intensity beam profiles ( $I$ versus $x, y)$ at the input plane of each GPL in the structure for the guided case; the scale-bar corresponds to $400 \mu \mathrm{m}$. 


\section{METHODS}

\section{A. Dynamics of circularly polarized waves through a uniform uniaxial medium}

Plane-wave light propagation along $z$ in a uniform uniaxial medium can be described by a two-component Jones vector $\boldsymbol{\psi}(z)$ (in the bra-ket notation $|\psi\rangle$ ). We then define a $2 \times 2$ transmission Jones matrix $\boldsymbol{T}(z, \theta)$ linking the vector $\boldsymbol{\psi}$ at the input plane $z=0$ with that at any given distance $z$, as a function of the angle $\theta$ formed by the optic axis with the $y$ axis. The two components of $\boldsymbol{\psi}$ represent the amplitudes of two orthogonal polarizations which define the chosen basis of the representation. Here in particular we employ the (LCP, RCP) basis. In this Letter, we adopt the notation $\hat{L}=(\hat{x}-i \hat{y}) / \sqrt{2}(\mathrm{LCP})$ and $\hat{R}=(\hat{x}+i \hat{y}) / \sqrt{2}(\mathrm{RCP})$, corresponding to the sourcepoint-of-view convention on $\mathrm{CP}$ states. The rotation operator $\boldsymbol{R}(\theta)$ around the $\hat{z}$ axis is diagonal in the CP basis and can be written as

$$
\boldsymbol{R}_{L R}(\theta)=\left(\begin{array}{cc}
e^{-i \theta} & 0 \\
0 & e^{i \theta}
\end{array}\right)
$$

with the subscript "LR" denoting the basis. The transmission matrix $\boldsymbol{T}_{L R}(z, \theta)$ has the form

$\boldsymbol{T}_{L R}(z, \theta)=\boldsymbol{R}_{L R}^{-1}(\theta) \cdot \boldsymbol{P}^{-1} \cdot\left(\begin{array}{cc}e^{i k_{0} n_{o} z} & 0 \\ 0 & e^{i k_{0} n_{e} z}\end{array}\right) \cdot \boldsymbol{P} \cdot \boldsymbol{R}_{L R}(\theta)$

where $\boldsymbol{P}=(1,1 ;-i, i) / \sqrt{2}$ is the matrix changing basis from $\{\hat{x}, \hat{y}\}$ to $\{\hat{L}, \hat{R}\}$. A straightforward derivation leads from Eq. (4) to Eq. (1).

Let us consider the solution for a purely circular polarization at the input, for e.g. $\psi_{R}(0)=1$ and $\psi_{L}(0)=0$. From Eq. (1) we get $\psi_{R}(z)=e^{i \bar{n} k_{0} z} \cos \left(\frac{\delta}{2}\right)$ and $\psi_{L}(z)=$ $-i e^{i \bar{n} k_{0} z} \sin \left(\frac{\delta}{2}\right) e^{2 i \theta}$. From the latter expression we can calculate the phase difference between two states corresponding to two different orientations for the optic axis, say $\theta_{1}$ and $\theta_{2}$, respectively. Following Pancharatnam's original concept ${ }^{25131}$, the phase delay $\Delta \phi\left(\theta_{1}, \theta_{2}\right)$ is

$$
\begin{aligned}
\Delta \phi\left(\theta_{1}, \theta_{2}\right) & =\arg \left[\left\langle\psi\left(\theta_{1}\right) \mid \psi\left(\theta_{2}\right)\right\rangle\right] \\
& =\arg \left[\cos ^{2}\left(\frac{\delta}{2}\right)+\sin ^{2}\left(\frac{\delta}{2}\right) e^{2 i\left(\theta_{2}-\theta_{1}\right)}\right]
\end{aligned}
$$

Expression (5) results in the transverse phase delay plotted in Fig. 1 $\mathbf{b}$ (dashed lines) in the homogeneous limit. When the optic axis distribution is flipped at $\Lambda / 2$, the phase delay can be easily obtained from Eq. (4) (solid lines in Fig. 1 b). When the optic axis distribution is sinusoidally modulated along $z$, the accumulated $\Delta \phi\left(\theta_{1}, \theta_{2}\right)$ can be numerically calculated partitioning the medium in several layers, each of them short enough to make the variations of $\theta$ negligible within each layer, as shown in Fig. 1b (solid lines with circles).

\section{B. Spin-dependent photonic potential}

In the paraxial limit (i.e., neglecting the longitudinal field components) and for small birefringence $(\Delta n \ll 1)$, the Maxwell equations for the electric field $\boldsymbol{E}=E_{x} \hat{x}+$ $E_{y} \hat{y}$ in two dimensions (i.e., with no field evolution across y) can be cast as

$$
\begin{aligned}
\frac{\partial^{2}}{\partial z^{2}}\left(\begin{array}{c}
E_{x} \\
E_{y}
\end{array}\right)= & -\frac{\partial^{2}}{\partial x^{2}}\left(\begin{array}{c}
E_{x} \\
E_{y}
\end{array}\right)+ \\
& -k_{0}^{2}\left(\begin{array}{cc}
\epsilon_{x x}(x, z) & \epsilon_{x y}(x, z) \\
\epsilon_{y x}(x, z) & \epsilon_{y y}(x, z)
\end{array}\right)\left(\begin{array}{c}
E_{x} \\
E_{y}
\end{array}\right)
\end{aligned}
$$

We would like to adopt now the slowly-varying-envelope approximation (SVEA) so as to obtain a simpler firstorder partial differential equation in the evolution coordinate $z$. SVEA is usually based on the presence of two very different spatial scales, that is a short scale of order $\lambda$ and a long scale given by the Rayleigh length $z_{R}=\pi \bar{n} w^{2} / \lambda$, where $w$ is the transverse spatial scale of the problem (the beam radius and/or the transverse spatial modulations of the medium). In our case, however, we also have an intermediate scale $\Lambda=\lambda / \Delta n$, with $\lambda \ll \Lambda \ll z_{R}$ for reasonable values of $\Delta n$ and $w$. It is therefore not convenient to apply the SVEA directly to Eq. (6), as the field components $E_{x}$ and $E_{y}$ undergo a relatively rapid evolution on scale $\Lambda$ because of birefringence. It is more convenient to switch first to the space-varying ordinary/extraordinary wave basis, with a rotation by the angle $\theta(x, z)$ around the $z$ axis. This basis change diagonalizes the effect of birefringence and hence allows one to adopt the SVEA in an optimal way, but at the same time it generates the space-varying geometric phases which affect the resulting slow-envelope dynamics. After applying the SVEA and neglecting higher-order terms in $\lambda / \Lambda$ and $\Lambda / z_{R}$, we obtain our final dynamical equations for the slowly-varying amplitudes (see Supplementary Material for a full derivation). We find in particular that the two components of the field can be decoupled from each other if one adopts the CP basis for the slow amplitudes (corresponding to the input states of the polarizations). The resulting dynamical equation for each of these amplitudes $A$ is

$$
\begin{aligned}
i \frac{\partial A}{\partial z}= & -\frac{1}{2 \bar{n} k_{0}} \frac{\partial^{2} A}{\partial x^{2}}+\frac{1}{4 \bar{n} k_{0}}\left[\left(\frac{d \Gamma}{d x}\right)^{2}+\frac{4 \pi^{2}}{\Lambda^{2}} \Gamma^{2}(x)\right] A \\
& \pm \frac{\pi}{\Lambda} \Gamma(x) A
\end{aligned}
$$

where $\pm=+$ for the amplitude $A$ corresponding to input $\mathrm{LCP}$ and $\pm=-$ for the amplitude corresponding to the input RCP. The guided modes of the system can then be obtained by setting $A(x, z)=e^{i \beta z} A(x, 0)$, with the effective propagation constant $\beta$ acting as an eigenvalue (hence $\Lambda \ll 1 / \beta$ must hold for consistency). Compared to the simpler model presented in the main text, the photonic potential contains a contribution related with the Kapitza effect ${ }^{17}$ due to both transverse $\left(\frac{d \Gamma}{d x}\right)$ and longitudinal $\left(\frac{d \sigma}{d z}\right)$ modulations of the optic axis orientation $\theta$ (see Supplementary Material for further details). 
Thus, neglecting the terms proportional to the square of $\Gamma$, the two circular polarizations perceive photonic potentials of the same transverse profile but opposite signs, as stated by Eq. (2). Equation (7) shows that a higherorder contribution due to a non-adiabatic modulation of the geometric phase (term in square brackets) acts in the same way on both components; when $w_{D}=5 \mu \mathrm{m}$ such term can be neglected for $\Gamma_{0}$ up to $360^{\circ}$, as apparent in Fig. 2a-b. The Kapitza term increases for narrower distributions of $\theta$ (Supplementary Fig. 1 shows how the trapping potential gets strongly distorted for $\Gamma_{0}=360^{\circ}$ when $\left.w_{D}=0.5 \mu \mathrm{m}\right)$.

\section{FDTD simulations}

For the FDTD numerical simulations we employed the open-source code MEEP ${ }^{32}$ to solve the full Maxwell equations in two dimensions, with no approximations. In all simulations we assumed a Gaussian shaped orientation of the optic axis across $x$ in the form $\Gamma(x)=$ $\Gamma_{0} \exp \left(-x^{2} / w_{D}^{2}\right)$. The excitation was a continuous-wave source launched in $x=z=0$ with a width of $3 \mu \mathrm{m}$ across $x$, turned on at $t=0$ and infinitely narrow across $z$. The modulated uniaxial medium was placed in $z_{0}=2$ $\mu \mathrm{m}$ with modulation $\sigma(z)=\sin \left[\frac{2 \pi \Delta n}{\lambda}\left(z-z_{0}\right)\right]$. The refractive indices $n_{o}$ and $n_{e}$ were taken equal to 1.5 and 1.7, respectively. The simulations confirm that an input $\mathrm{RCP}$ is confined in the anisotropic structure, whereas an LCP input is expelled towards the edges (Supplementary Fig. 3). The polarization of the simulated confined wave undergoes small variations in the transverse plane (Supplementary Fig. 2) and is not perfectly periodic along $z$. Such small discrepancies between simulations and our analytic theory are clearly due to higher-order terms in $\lambda / \Lambda$ and $\Lambda / z_{R}$ which have been neglected in the analytic theory. For $w_{D}=5 \mu \mathrm{m}$ light confinement improves with the maximum rotation $\Gamma_{0}$ (Supplementary Fig. 3); however, for $\Gamma_{0}=90^{\circ}$ even in the defocusing case a small amount of power is trapped on axis owing to the higher order terms (Kapitza effect) previously neglected in Eq. (7). Further simulations show how light trapping/repulsion both increase as the width $w_{D}$ gets smaller, in agreement with theory (Supplementary Fig. 5). We also studied numerically the effects of shifting $\sigma(z)$ by changing $z_{0}$, obtaining a perfect agreement with theory. In fact, when $z_{0}=z_{\text {coh }} / 2$ the two polarization states exchange their roles, that is, LCP at the input gets trapped whereas a launched RCP undergoes defocusing (Supplementary Fig. 6); when $z_{0}=z_{\text {coh }} / 4$ the beam evolution does not depend on the input polarization, with power equally shared by confined and radiated modes. These results demonstrate that the PB-phase waveguide infringes translational symmetry along the propagation axis, at variance with standard (TIR or GRIN) waveguides. Finally, we numerically ascertained the role of small mismatches between the modulation period $\Lambda$ and the length $z_{\text {coh }}$, an important issue in actual implementa- tions of the proposed waveguides. Keeping all the parameters fixed except for $\Lambda$, the global behavior of the two polarization states, i.e., LCP defocused and RCP guided, is robust against $\Lambda$ variations up to $50 \%$.

\section{Fabrication of the geometric phase lenses}

Geometric phase lenses and similar PBOEs can be realized with a variety of techniques and materials 20 22 33/34. Liquid crystals ${ }^{21}$ and liquid crystal polymers 33 are the most suitable for visible and near infrared illumination. Our GPLs were fabricated using polarization holography in combination with photo-alignment of nematic liquid crysta 35 . Planar cells were realized with two glass substrates held parallel at a separation of $6 \mu \mathrm{m}$, previously coated with a convenient photoaligning surfactant 35 . Such substrates were exposed to collimated ultraviolet light with an inhomogeneous distribution of the linear polarization state. The polarization pattern was realized by coaxial superposition of two beams (473 nm diodepumped solid-state laser) with orthogonal circular polarizations and various phase-front curvatures. The interference of such beams, rather than an intensity modulation, produces a pattern of linear polarizations, with the angle of the polarization plane proportional to the point-wise phase difference between the beams. After exposure of the coated glass slides, nematic liquid crystals (mixture E7 from Merck) were introduced in the cell by capillarity and aligned with a correspondingly inhomogeneous orientation of the optic axis (molecular director). The required half-wave phase retardation of the GPLs was finely adjusted by applying a $10 \mathrm{kHz}$ square-wave electric voltage $(\approx 2.5 \mathrm{~V}$ peak-peak $)$ as in other liquidcrystal-based PBOEs 36 . Each GPL can also be optically "switched off" by applying the voltage giving full-wave retardation $(\approx 4.0 \mathrm{~V})$. Five GPLs were fabricated, with focal lengths 15.17, 15.98, 14.83, 15.17 and $15.69 \pm 0.01$ $\mathrm{cm}$, respectively, for light of wavelength $532 \mathrm{~nm}$. The dispersion of the focal length values is due to the imperfect repeatability of the exposure conditions and insufficient stability of the fabrication setup.

\section{E. Beam characterization within the structure}

In order to reconstruct the beam parameters inside the GPL structure, the propagating beam at $532 \mathrm{~nm}$ was sampled by a CCD camera placed at various propagation distances between the lenses. Moreover, to improve the measurement accuracy of the local beam parameters, additional beam profiles were collected at given distances from each lens. This was accomplished by either switching off the GPLs following the one under measurement or by physically removing the remaining lenses from the sequence. The beam radii $w(z)$ were obtained through Gaussian fits of the acquired profile images and used to reconstruct the modal parameters within and at 
the output of the waveguide. The obtained radius evolution was then compared with the theoretical predictions from ABCD Gaussian propagation and numerical simulations. A more realistic description of the beam was obtained with a non-unitary beam-quality factor $M^{2}$ of the confined beams after each lens $\left(M_{1}^{2}=1.05 \pm 0.01\right.$, $M_{2}^{2}=1.18 \pm 0.02, M_{3}^{2}=1.19 \pm 0.01, M_{4}^{2}=1.15 \pm 0.04$, and $M_{5}^{2}=1.32 \pm 0.04$, uncertainties at $95 \%$ confidence level), suitably modifying the propagation equations 37 for the simulations. The gradual increase of the $M^{2}$ parameter after each step of the discrete structure can be ascribed to degradation of the beam profile (as visible in Fig. 3p) due to the noisy patterns of the GPLs.

\section{Acknowledgments and author contributions}

The work in Naples was partly supported by the $7^{t h}$ Framework Programme of the European Commission, within the Future Emerging Technologies program, under grant No. 255914, PHORBITECH. A.A. and G.A. thank the Academy of Finland for financial support through the FiDiPro grant no. 282858. C.P.J. gratefully acknowledges Fundação para a Ciência e a Tecnologia, POPH-QREN and FSE (FCT, Portugal) for the fellowship SFRH/BPD/77524/2011.

This work was jointly conceived by A.A., C.P.J., G.A. and L.M.; S.S. designed and carried out the experiment, with the help and supervision of B.P., E.S. and L.M.; A.A. and C.P.J. developed the theory and performed the numerical simulations, with the help and supervision of G.A.; all authors discussed the results and contributed to the manuscript.
1 A. W. Snyder and J. D. Love. Optical Waveguide Theory. Chapman and Hall, New York, 1983.

2 J. D. Joannopoulos, P. R. Villeneuve, and S. H. Fan. Photonic crystals: Putting a new twist on light. Nature, 386:143-149, 1997.

3 J. C. Knight. Photonic crystal fibres. Nature, 424:847-851, 2003.

4 P. Russell. Photonic crystal fibers. Science, 299:358-362, 2003.

5 P. Yeh and A. Yariv. Bragg reflection waveguides. Opt. Commun., 19:427-430, 1976.

${ }^{6}$ R. Y. Chiao and Yong-Shi Wu. Manifestations of Berry's topological phase for the photon. Phys. Rev. Lett., 57:933, 1986.

7 F. D. M. Haldane. Path dependence of the geometric rotation of polarization in optical fibers. Opt. Lett., 11:730-732, 1986.

${ }^{8}$ M. V. Berry. The adiabatic phase and Pancharatnam's phase for polarized light. J. Mod. Opt., 34:1401-1407, 1987.

9 R. Bhandari. Polarization of light and topological phases. Phys. Rep., 281(1):1-64, 1997.

10 D. Andrews. Structured light and its applications. Academic Press, London, 2008.

11 A. M Yao and M. J. Padgett. Orbital angular momentum: origins, behavior and applications. Adv. Opt. Photon., 3:161-204, 2011.

12 V. R. Almeida, Q. Xu, C. A. Barrios, and M. Lipson. Guiding and confining light in void nanostructure. Opt. Lett., 29(11):1209-1211, 2004.

13 J.-C. Weeber, Y. Lacroute, and A. Dereux. Optical nearfield distributions of surface plasmon waveguide modes. Phys. Rev. B, 68:115401, 2003.

14 A. Yariv, Y. Xu, R. K. Lee, and A. Scherer. Coupledresonator optical waveguide: a proposal and analysis. Opt. Lett., 24(11):711-713, 1999.

15 Q. Lin and S. Fan. Light guiding by effective gauge field for photons. Phys. Rev. X, 4:031031, 2014.

16 O. Cohen, B. Freedman, J. W. Fleischer, M. Segev, and D. N. Christodoulides. Grating-mediated waveguiding.
Phys. Rev. Lett., 93:103902, 2004.

17 A. Alberucci, L. Marrucci, and G. Assanto. Light confinement via periodic modulation of the refractive index. New J. Phys., 15(8):083013, 2013.

18 M. Peccianti, C. Conti, G. Assanto, A. De Luca, and C. Umeton. Routing of anisotropic spatial solitons and modulational instability in liquid crystals. Nature, 432:733-737, 2004.

19 K. Y. Bliokh, F. J. Rodriguez-Fortuno, F. Nori, and A. V. Zayats. Spin-orbit interactions of light. Nature Photon., 9:796-808, 2015.

20 Z. Bomzon, V. Kleiner, and E. Hasman. PancharatnamBerry phase in space-variant polarization-state manipulations with subwavelength gratings. Opt. Lett., 26(18):1424-1426, 2001.

21 L. Marrucci, C. Manzo, and D. Paparo. Optical spin-toorbital angular momentum conversion in inhomogeneous anisotropic media. Phys. Rev. Lett., 96:163905, 2006.

22 S. Slussarenko, A. Murauski, T. Du, V. Chigrinov, L. Marrucci, and E. Santamato. Tunable liquid crystal q-plates with arbitrary topological charge. Opt. Express, 19:40854090, 2011.

23 N. Yu and F. Capasso. Flat optics with designer metasurfaces. Nature Mater., 13:139-150, 2014.

24 D. Lin, P. Fan, E. Hasman, and M. L. Brongersma. Dielectric gradient metasurface optical elements. Science, 345:298-302, 2014.

25 S. Pancharatnam. Generalized theory of interference, and its applications. Proc. Indian Acad. Sci. A, 44(5):03700089, 1956.

${ }^{26}$ C. Lin, L. G. Cohen, and H. Kogelnik. Optical-pulse equalization of low-dispersion transmission in single-mode fibers in the 1.3-1.7- $\mu \mathrm{m}$ spectral region. Opt. Lett., 5(11):476478, 1980 .

27 J. A. Armstrong, N. Bloembergen, J. Ducuing, and P. S. Pershan. Interactions between light waves in a nonlinear dielectric. Phys. Rev., 127:1918-1939, 1962.

28 E Hasman, V Kleiner, G Biener, and A Niv. Polarization dependent focusing lens by use of quantized Pancharatnam-Berry phase diffractive optics. Appl. Phys. 
Lett., 82(3):328-330, 2003.

29 F. S. Roux. Geometric phase lens. J. Opt. Soc. Am. A, 23(2):476-482, 2006.

30 L. Marrucci, C. Manzo, and D. Paparo. PancharatnamBerry phase optical elements for wavefront shaping in the visible domain: switchable helical modes generation. Appl. Phys. Lett., 88:221102, 2006.

31 M. V. Berry. Pancharatnam, virtuoso of the Poincaré sphere: an appreciation. Current Science, 67:220-223, 1994.

32 A. F. Oskooi, D. Roundy, M. Ibanescu, P. Bermel, J. D. Joannopoulos, and S. G. Johnson. MEEP: A flexible freesoftware package for electromagnetic simulations by the FDTD method. Computer Phys. Commun., 181:687-702, 2010.

33 S. Nersisyan, N. Tabiryan, D. M. Steeves, and B. R. Kimball. Fabrication of liquid crystal polymer axial waveplates for UV-IR wavelengths. Opt. Express, 17(14):11926-11934, 2009.

34 C. N. Alexeyev. Circular array of anisotropic fibers: A discrete analog of a $q$ plate. Phys. Rev. A, 86:063830, 2012.

35 V. G. Chigrinov, V. M. Kozenkov, and H.-S. Kwok. Photoalignment of Liquid Crystalline Materials: Physics and Applications. Wiley Publishing, 2008.

36 B. Piccirillo, V. D’Ambrosio, S. Slussarenko, L. Marrucci, and E. Santamato. Photon spin-to-orbital angular momentum conversion via an electrically tunable q-plate. Appl. Phys. Lett., 97(5):241104, 2010.

37 H. Sun. Thin lens equation for a real laser beam with weak lens aperture truncation. Opt. Eng., 37(11):29062913, 1998. 


\section{SUPPLEMENTARY INFORMATION}

\section{S1. LIGHT PROPAGATION IN A PERIODIC SYSTEM ENCOMPASSING A ROTATION OF THE OPTIC AXIS IN THE TRANSVERSE PLANE}

We consider light propagation in inhomogeneous anisotropic dielectrics, in particular uniaxials; nonetheless, our results can be readily generalized to biaxial crystals. We take a medium whose dielectric properties vary across the transverse coordinate $x$ owing exclusively to a rotation of the optic axis in the plane $x y$ orthogonal to the propagation coordinate $z$. We consider finite wavepackets with wavevector parallel to $\hat{z}$; hence, corresponding to electric fields oscillating orthogonally and parallel to the optic axis, respectively, the two independent eigenvalues $\epsilon_{\perp}$ and $\epsilon_{\|}$of the relative permittivity tensor are constant in space. We define the birefringence $\Delta n=\sqrt{\epsilon_{\|}}-\sqrt{\epsilon_{\perp}}=n_{e}-n_{o}$ and assume that the distribution of the optic axis is purely planar and transverse to $\hat{z}$, such that at each $z$ the rotation can be described by a standard 2D operator acting in $x y$ :

$$
\boldsymbol{R}(\theta)=\left(\begin{array}{cc}
\cos \theta & \sin \theta \\
-\sin \theta & \cos \theta
\end{array}\right)
$$

where the angle $\theta$ is defined with respect to the $y$ axis of a Cartesian reference system in the laboratory frame, with $\theta=0$ corresponding to a dielectric permittivity $\epsilon_{\|}$for electric fields along $y$. We study forward propagating light waves in the presence of a periodic modulation of $\theta$ along $z$. To this extent we set

$$
\theta(x, z)=\sigma(z) \Gamma(x)=\left(\sum_{p=-\infty}^{\infty} \sigma_{p} e^{\frac{i 2 \pi p z}{\Lambda}}\right) \Gamma(x),
$$

with $\sigma(z)$ a function periodic with $\Lambda$ and $\Gamma(x)$ the transverse distribution of optic axis orientation, the latter being uniform across $y$. Hereafter, we examine a purely sinusoidal modulation $\sigma_{p}=\sigma_{1} \delta_{1, p}+\sigma_{-1} \delta_{-1, p}$, with $\delta_{p, p^{\prime}}$ the Kronecker's delta and $\sigma_{-1}=\sigma_{1}^{*}$ in order for $\sigma$ to be real valued. Moreover, we deal with the case $\Lambda=\lambda / \Delta n$ in which resonant effects are expected to occur.

In the paraxial limit (neglecting longitudinal field components) and for small birefringence $(\Delta n \ll 1)$, Maxwell equations in two dimensions (i.e., no field evolution across y) can be cast as

$$
\frac{\partial^{2}}{\partial z^{2}}\left(\begin{array}{c}
E_{x} \\
E_{y}
\end{array}\right)=-\frac{\partial^{2}}{\partial x^{2}}\left(\begin{array}{c}
E_{x} \\
E_{y}
\end{array}\right)-k_{0}^{2}\left(\begin{array}{cc}
\epsilon_{x x}(x, z) & \epsilon_{x y}(x, z) \\
\epsilon_{y x}(x, z) & \epsilon_{y y}(x, z)
\end{array}\right)\left(\begin{array}{c}
E_{x} \\
E_{y}
\end{array}\right)
$$

where $\boldsymbol{E}=E_{x} \hat{x}+E_{y} \hat{y}$ is the electric field.

In order to investigate the propagation of an electromagnetic (optical) wave in such a system, we make use of the transformation $\varphi=\boldsymbol{R} \cdot \boldsymbol{E}$ and write $\varphi=\left(E_{o} ; E_{e}\right)$, with $E_{o}$ and $E_{e}$ the pointwise ordinary and extraordinary polarization components of the electric field, respectively. In the rotated reference system the two-dimensional dielectric tensor is diagonal, specifically $\boldsymbol{\epsilon}_{D}=\left(\epsilon_{\perp}, 0 ; 0, \epsilon_{\|}\right)$; after setting $\boldsymbol{T}=(0,-1 ; 1,0)$, Eq. (S3) becomes

$$
\frac{\partial^{2} \boldsymbol{\varphi}}{\partial z^{2}}+2 \frac{\partial \theta}{\partial z} \boldsymbol{T} \cdot \frac{\partial \boldsymbol{\varphi}}{\partial z}=-\left\{\frac{\partial^{2} \boldsymbol{\varphi}}{\partial x^{2}}-\left[\left(\frac{\partial \theta}{\partial x}\right)^{2}+\left(\frac{\partial \theta}{\partial z}\right)^{2}\right] \boldsymbol{\varphi}\right\}-k_{0}^{2} \boldsymbol{\epsilon}_{D} \cdot \boldsymbol{\varphi}-\left(\frac{\partial^{2} \theta}{\partial x^{2}}+\frac{\partial^{2} \theta}{\partial z^{2}}\right) \boldsymbol{T} \cdot \boldsymbol{\varphi}-2 \frac{\partial \theta}{\partial x} \boldsymbol{T} \cdot \frac{\partial \boldsymbol{\varphi}}{\partial x} .
$$

Equation (S4) shows that a scalar potential proportional to $(\partial \theta / \partial x)^{2}+(\partial \theta / \partial z)^{2}$ acts on both field components. Additionally, other terms (containing the operator $\boldsymbol{T}$ ) couple ordinary and extraordinary polarizations: due to the rotation of the optic axis, the ordinary and extraordinary components are no longer independent, but can exchange energy during propagation. Noteworthy, it is apparent from Eq. (S4) that no refractive index gradients affect propagation as the latter is entirely governed by the power exchange between ordinary and extraordinary components, in turn leading to a complex evolution of the overall polarization state.

In the slowly varying envelope approximation (SVEA), we first set $E_{o}=\psi_{o}(x, z) e^{i k_{0} n_{o} z}$ and $E_{e}=\psi_{e}(x, z) e^{i k_{0} n_{e} z}$ in Eq. (S4). Exploiting the system periodicity in $z$ we then introduce the following further transformation:

$$
\psi_{j}(x, z)=A_{j}(x, z) B_{j}(x, z) \quad(j=e, o),
$$

where $A_{j}(x, z)$ are the slow-varying envelopes for the two wave components and $B_{j}(x, z)$ are periodic functions of $z$ accounting for the effect of the medium modulations with period $\Lambda$. It is convenient to write the latter ones in an 
exponential form as follows:

$$
B_{j}(x, z)=e^{i \sum_{p=-\infty}^{\infty} \int \beta_{p}^{(j)}(x, z) d z}=\exp \left[\sum_{p=-\infty}^{\infty} \frac{\Lambda}{2 \pi p} \beta_{p}^{(j)}(x) e^{\frac{i 2 \pi p z}{\Lambda}}\right]
$$

where we set $\beta_{p}^{(j)}(x, z)=\beta_{p}^{(j)}(x) e^{\frac{i 2 \pi p z}{\Lambda}}$ and the term $p=0$ must be excluded from the sum. Inserting these ansatzes in Eq. (S4) and expanding in powers of $\Lambda$ all terms one obtains a hierarchy of coupled equations for the amplitudes $A_{j}(x, z)$ and the coefficients $\beta_{p}^{(j)}(x)$. Neglecting from these equations all terms with powers of the period $\Lambda$ equal to or larger than 1 and higher-order terms in $\lambda / \Lambda$ (which is equal to $\Delta n$ at resonance), we obtain the following coupled equations for the slow amplitudes:

$$
\begin{gathered}
i \frac{\partial A_{o}}{\partial z}=-\frac{1}{2 \bar{n} k_{0}} \frac{\partial^{2} A_{o}}{\partial x^{2}}+\frac{1}{2 \bar{n} k_{0}}\left[\overline{\sigma^{2}(z)}\left(\frac{d \Gamma}{d x}\right)^{2}+\Gamma^{2} \overline{\left(\frac{d \sigma}{d z}\right)^{2}}\right] A_{o}+\frac{2 \pi}{\Lambda} \sigma_{-1} \Gamma(x) A_{e} \\
i \frac{\partial A_{e}}{\partial z}=-\frac{1}{2 \bar{n} k_{0}} \frac{\partial^{2} A_{e}}{\partial x^{2}}+\frac{1}{2 \bar{n} k_{0}}\left[\overline{\sigma^{2}(z)}\left(\frac{d \Gamma}{d x}\right)^{2}+\Gamma^{2} \overline{\left(\frac{d \sigma}{d z}\right)^{2}}\right] A_{e}+\frac{2 \pi}{\Lambda} \sigma_{1} \Gamma(x) A_{0}
\end{gathered}
$$

where $\overline{m(z)}=\frac{1}{\Lambda} \int_{0}^{\Lambda} m(z) d z$ and $\bar{n}=\left(n_{e}+n_{o}\right) / 2$.

After inspection of Eqs. $\mathrm{S} 7 \mathrm{~S} 8 \mathrm{n}$, three salient terms stand out:

- a Kapitza-like potential, proportional to the squared transverse derivative of the distribution $\Gamma$ of optic axis orientation; its magnitude is modulated by the mean square of the periodic modulation $\sigma(z)$;

- an effective transverse potential with profile proportional to $\Gamma^{2}$; its magnitude is modulated by the mean square of the longitudinal derivative of the periodic modulation $\sigma(z)$;

- a phase-matched coupling between ordinary and extraordinary waves via the fundamental harmonics $\sigma_{ \pm 1}$ of the periodic modulation.

The equations $[\mathrm{S} 7 \mathrm{~S} 8 \mathrm{~s}$ can be recast in a more compact form as

$$
i \frac{\partial \boldsymbol{A}}{\partial z}=\boldsymbol{L}_{\mathrm{ISO}} \cdot \boldsymbol{A}+\boldsymbol{L}_{\mathrm{ANI}} \cdot \boldsymbol{A},
$$

where $\boldsymbol{A}=\left(A_{o} ; A_{e}\right)$ and we introduced the isotropic (matrix) operator

$$
\boldsymbol{L}_{\mathrm{ISO}}=\frac{1}{2 \bar{n} k_{0}}\left\{-\frac{\partial^{2}}{\partial x^{2}}+\left[\overline{\sigma^{2}}\left(\frac{d \Gamma}{d x}\right)^{2}+\Gamma^{2} \overline{\left(\frac{d \sigma}{d z}\right)^{2}}\right]\right\} \boldsymbol{I},
$$

and the anisotropic operator

$$
\boldsymbol{L}_{\mathrm{ANI}}=\frac{2 \pi}{\Lambda} \Gamma(x)\left(\begin{array}{cc}
0 & \sigma_{-1} \\
\sigma_{1} & 0
\end{array}\right) .
$$

The presence of $\boldsymbol{L}_{\mathrm{ANI}}$ in Eq. (S9) accounts for the power exchange between ordinary and extraordinary components.

Let us now take $\sigma(z)=\sin \left(\frac{2 \pi z}{\Lambda}\right)$, that is $\sigma_{1}=-i / 2$ and $\sigma_{-1}=i / 2$; then $\boldsymbol{L}_{\mathrm{ANI}}=-i \frac{\pi}{\Lambda} \Gamma(x) \boldsymbol{T}$ and its eigenvalues are $\pm i$ with the two circular polarizations $(1 ; \pm i) / \sqrt{2}$ for eigenvectors (plus and minus correspond to RCP and LCP in our convention, respectively). Therefore, when the optic axis is modulated along $z$ with period equal to the beat length, the localized wave solutions are circularly polarized. By using the transformation

$$
\boldsymbol{A}_{o e}=\boldsymbol{P} \cdot \boldsymbol{A}_{L R}=\frac{1}{\sqrt{2}}\left(\begin{array}{cc}
1 & 1 \\
-i & i
\end{array}\right)\left(\begin{array}{c}
A_{L} \\
A_{R}
\end{array}\right),
$$

the system of equations separates into two independent scalar equations

$$
\begin{aligned}
& i \frac{\partial A_{L}}{\partial z}=-\frac{1}{2 \bar{n} k_{0}} \frac{\partial^{2} A_{L}}{\partial x^{2}}+\frac{1}{4 \bar{n} k_{0}}\left[\left(\frac{d \Gamma}{d x}\right)^{2}+\frac{4 \pi^{2}}{\Lambda^{2}} \Gamma^{2}(x)\right] A_{L}+\frac{\pi}{\Lambda} \Gamma(x) A_{L}, \\
& i \frac{\partial A_{R}}{\partial z}=-\frac{1}{2 \bar{n} k_{0}} \frac{\partial^{2} A_{R}}{\partial x^{2}}+\frac{1}{4 \bar{n} k_{0}}\left[\left(\frac{d \Gamma}{d x}\right)^{2}+\frac{4 \pi^{2}}{\Lambda^{2}} \Gamma^{2}(x)\right] A_{R}-\frac{\pi}{\Lambda} \Gamma(x) A_{R},
\end{aligned}
$$


where we used $\overline{\sigma^{2}(z)}=0.5$ and $\overline{(d \sigma / d z)^{2}}=2 \pi^{2} / \Lambda^{2}$. The guided eigenmodes are then defined by setting $A_{i}(x, z)=$ $e^{i \beta_{0} z} A_{i}(x, 0)$, where $\beta_{0}$ is the propagation constant. The problem is then transformed into a standard eigenvalue problem, with the following equations:

$$
\begin{aligned}
& -2 k_{0} \bar{n} \beta_{0} A_{L}=-\frac{\partial^{2} A_{L}}{\partial x^{2}}+\left[\frac{1}{2}\left(\frac{d \Gamma}{d x}\right)^{2}+\frac{2 \pi^{2}}{\Lambda^{2}} \Gamma^{2}(x)\right] A_{L}+\frac{2 k_{0} \bar{n} \pi}{\Lambda} \Gamma(x) A_{L}, \\
& -2 k_{0} \bar{n} \beta_{0} A_{R}=-\frac{\partial^{2} A_{R}}{\partial x^{2}}+\left[\frac{1}{2}\left(\frac{d \Gamma}{d x}\right)^{2}+\frac{2 \pi^{2}}{\Lambda^{2}} \Gamma^{2}(x)\right] A_{R}-\frac{2 k_{0} \bar{n} \pi}{\Lambda} \Gamma(x) A_{R},
\end{aligned}
$$

Equations $(\mathrm{S} 15)$ and $(\mathrm{S} 16)$ are valid for left (LCP) and right (RCP) circularly polarized wavepackets at the input interface, respectively. It is noteworthy that RCP and LCP interchange roles if the modulation $\sigma(z)$ is shifted by half a period, in agreement with the intuitive picture provided in the main text.

\section{A. Polarization-dependent effective index well}

In standard $(1+1) \mathrm{D}$ graded-index waveguides, a generic transverse-electric mode $u$ satisfies $-2 k_{0} \bar{n} \kappa u=-\frac{\partial^{2} u}{\partial x^{2}}-$ $k_{0}^{2} \Delta n^{2} u$, with $\kappa$ the propagation constant. Thus, from Eqs. S15 S16 it is apparent that the two circular polarizations perceive the effective photonic potential $V_{\text {eff }}=-k_{0} \Delta n_{\text {eff }}^{2} /(2 \bar{n})$ with the index distributions $\Delta n_{\text {eff }}^{2}(x)$ given by

$$
\begin{gathered}
\Delta n_{\mathrm{eff}, L C P}^{2}=-\frac{2 \bar{n}}{k_{0}} V_{\mathrm{eff}, L C P}=-\frac{1}{k_{0}^{2}}\left[\frac{1}{2}\left(\frac{d \Gamma}{d x}\right)^{2}+\frac{2 \pi^{2}}{\Lambda^{2}} \Gamma^{2}(x)\right]+\bar{n} \frac{\lambda}{\Lambda} \Gamma(x), \\
\Delta n_{\mathrm{eff}, R C P}^{2}=-\frac{2 \bar{n}}{k_{0}} V_{\mathrm{eff}, R C P}=-\frac{1}{k_{0}^{2}}\left[\frac{1}{2}\left(\frac{d \Gamma}{d x}\right)^{2}+\frac{2 \pi^{2}}{\Lambda^{2}} \Gamma^{2}(x)\right]-\bar{n} \frac{\lambda}{\Lambda} \Gamma(x),
\end{gathered}
$$

The polarization independent term between square brackets in Eqs. S17.S18 is a Kapitza-like equivalent photonic potential stemming from transverse and longitudinal modulation of the rotating optic axis. Since this photonic potential is independent from $k_{0}=2 \pi / \lambda$, by itself it would support guided modes with wavelength-independent profile ${ }^{38}$. The last terms on the RHS of Eqs. (S17) S18, with opposite signs as wave handedness reverses, are responsible for the strong dependence of light evolution on input polarization: the periodic rotation of the optic axis along $z$ allows for an accumulation of the Berry phase during propagation, leading to the appearance of a potential proportional to $\Gamma$. Moreover, since phase-matching requires $\Lambda=\lambda / \Delta n$, such potential is directly proportional to the medium birefringence.

\section{B. Bell-shaped orientation distribution of the optic axis}

Hereafter we make explicit reference to optical frequencies. Nevertheless, since Maxwell equations are invariant when dividing all the length scales by a given factor and multiplying the frequency by the same factor, our results clearly hold valid regardless the electromagnetic band.

Supplementary Fig. S1 shows the effective index well $-\Delta n_{\text {eff }}^{2}$ (sign-inverted, so light is attracted by the dip) as computed from Eqs. (S17) S18) when the transverse distribution of the orientation angle $\Gamma(x)$ is bell-shaped and centered in $x=0$. We assumed $\Gamma(x)=\Gamma_{0} \exp \left(-x^{2} / w_{D}^{2}\right)$, with $\Gamma_{0}$ and $w_{D}$ the maximum orientation angle and the width of the distribution, respectively. Using this simple ansatz we can address the role of each term in the effective index well, Eqs. (S17 S18). The term proportional to $(d \Gamma / d x)^{2}$ is a Kapitza-like term: when acting alone it yields quasi-modes, as detailed in Ref. ${ }^{[17}$. This contribution to the index landscape, proportional to $w_{D}^{-2}$, increases as the angle distribution becomes narrower. Supplementary Fig. S1 illustrates two examples for $w_{D}=0.5 \mu \mathrm{m}$ and $w_{D}=15$ $\mu \mathrm{m}$, respectively: the Kapitza term becomes quite relevant for $w_{D}=0.5 \mu \mathrm{m}$ and large $\Gamma_{0}$, with the appearance of two local maxima, symmetrically placed with respect to the axis $x=0$. The term proportional to $\Gamma^{2}(x)$ gives rise to anti-guidance, i.e. light repulsion from the symmetry axis. The term is proportional to the square of the birefringence $\Delta n$, thus dominates for large anisotropies.

Finally, the last term breaks the degeneracy between the two opposite circular polarizations. This term contributes with opposite signs to the overall potential acting on RCP and LCP waves, respectively: in the absence of other contributions, when the RCP (LCP) wave is confined, the LCP (RCP) is repelled from the region close to the symmetry axis $x=0$, i.e., it diffracts faster than in a homogeneous medium. 

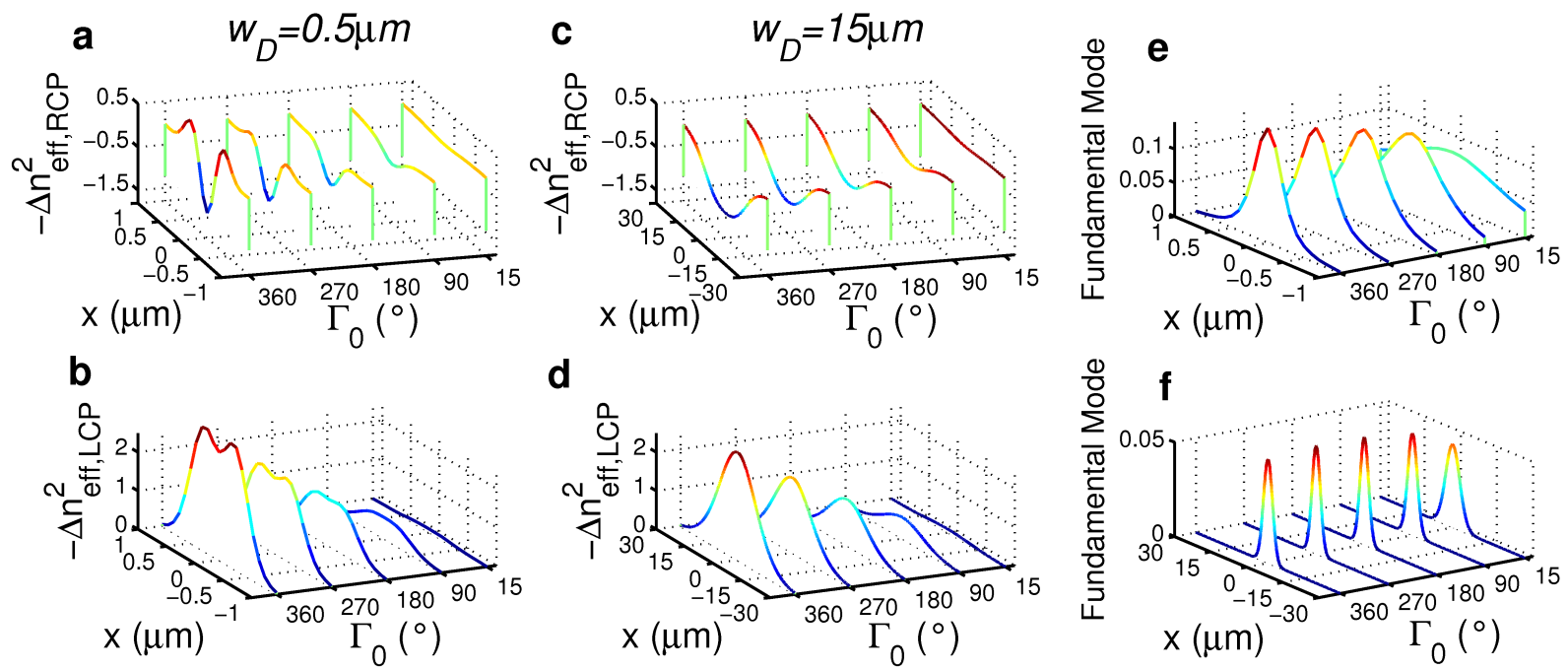

FIG. S1. a-d Transverse profile of the sign-inverted effective index distribution, proportional to the photonic potential $V_{\text {eff }, L C P / R C P}$ versus maximum reorientation angle $\Gamma_{0}$ for two different widths $(\mathbf{a}-\mathbf{b}) w_{D}=0.5 \mu \mathrm{m}$ and $(\mathbf{c}-\mathbf{d}) w_{D}=15 \mu \mathrm{m}$ of the transverse angular distribution. Input RCP waves are trapped in $\mathbf{a}, \mathbf{c}$ and input LCP waves undergo defocusing in $\mathbf{b}$, $\mathbf{d}$, respectively, in agreement with the chosen initial section. e-f Profiles of the fundamental guided (RCP) mode when (e) $w_{D}=0.5 \mu \mathrm{m}$ and $(\mathbf{f}) w_{D}=15 \mu \mathrm{m}$. Here $\lambda=1 \mu \mathrm{m}, n_{o}=1.5$ and $n_{e}=1.7$.

\section{Finite-Difference-Time-Domain numerical simulations of the guiding case}

In the numerical simulations we employed the open-source FDTD code named MEEP ${ }^{32}$ to solve the full Maxwell equations in two dimensions, assuming a Gaussian orientation of the optic axis across $x$, as above. The excitation was a continuous-wave source of wavelength $1 \mu \mathrm{m}$ turned on at $t=0$, infinitely narrow across $z$ and launched in $x=z=0$. The source had a Gaussian profile of width $3 \mu \mathrm{m}$ across $x$ and was point-like along $z$. The modulated uniaxial medium was placed in $z>z_{0}=2 \mu \mathrm{m}$ with modulation $\sigma(z)=\sin \left[\frac{2 \pi \Delta n}{\lambda}\left(z-z_{0}\right)\right]$. The refractive indices $n_{o}$ and $n_{e}$ were taken equal to 1.5 and 1.7 , respectively.

Supplementary Fig. S2 illustrates the propagation of an RCP wave input when $w_{D}=5 \mu \mathrm{m}$ and $\Gamma_{0}=15^{\circ}$, corresponding to a guiding potential (see Fig. 2 in the main text). As predicted, the natural diffractive spreading is compensated for by the effective waveguide resulting from Berry phase accumulation. At distances far enough from the input, in the stationary regime, the wavepacket acquires a periodic spatial distribution of its polarization and a nearly invariant profile. The polarization is generally elliptical; its trend can be examined by taking a single period $\lambda / \Delta n$ far enough from the input so that radiation (from imperfect coupling) is negligible. The polarization at the input is RCP, then it starts decreasing ellipticity; at about a quarter period it becomes linear, then elliptical again but with opposite handedness; at half period is nearly LCP, in excellent agreement with the theory. In the following half-period the polarization evolves in a similar manner, going from LCP to RCP. The polarization rotation versus propagation strictly resembles the behavior of a plane wave because it originates from the different phase velocities of the two linear eigenfield carriers (with $e^{i k_{0} n_{o} z}$ and $e^{i k_{0} n_{e} z}$, respectively).

Supplementary Fig. S3 compares the intensity evolution of propagating LCP and RCP wavepackets. In agreement with theory, one input polarization is subject to trapping, the other to a repulsive potential expelling light from the region around $x=0$. The strength of either potentials increases with the maximum rotation $\Gamma_{0}$ : in the trapping case the waveguide eventually becomes multi-modal, as indicated by the appearance of breathing versus propagation; in the repulsive case the beam divergence increases with $\Gamma_{0}$. For $\Gamma_{0} \geq 90^{\circ}$ the polarization of the confined beam continues to oscillate along $z$, but in the presence of higher harmonics. In fact, in Eq. (S6) the functions $\beta_{p}(x, z)$ with $p \geq 1$ must be accounted for and correspond to shorter periodicity in both beam profile and polarization. Simultaneously, a higher-order contribution proportional to $\Lambda^{2}\left(\frac{d \Gamma}{d x} \frac{d^{2} \Gamma}{d x^{2}}\right)^{2}$ appears in the overall photonic potential of the isotropic operator $\boldsymbol{L}_{\mathrm{ISO}}$ defined by Eq. S10 ${ }^{39}$. Accordingly, even in the defocusing case a small portion of the wavepacket is trapped on-axis around $x=0$ for $\Gamma_{0}=90^{\circ}$, as visible in Fig. S3. Owing to the additional modulating terms $\beta_{p}^{(j)}(x)$, further increases in $\Gamma_{0}$ (not shown) infringe the validity of Eqs. (S15 S16), leading to the generation of three-peaks for both input polarizations as well as appreciable changes of polarization state across $x$ in the guided case.

Supplementary Fig. S4 shows how power is distributed between the two components $E_{x}$ and $E_{y}$ when light is guided: $\left|E_{x}\right|^{2}$ and $\left|E_{y}\right|^{2}$ distributions in the plane $x z$ essentially coincide. The overall intensity is computed as 

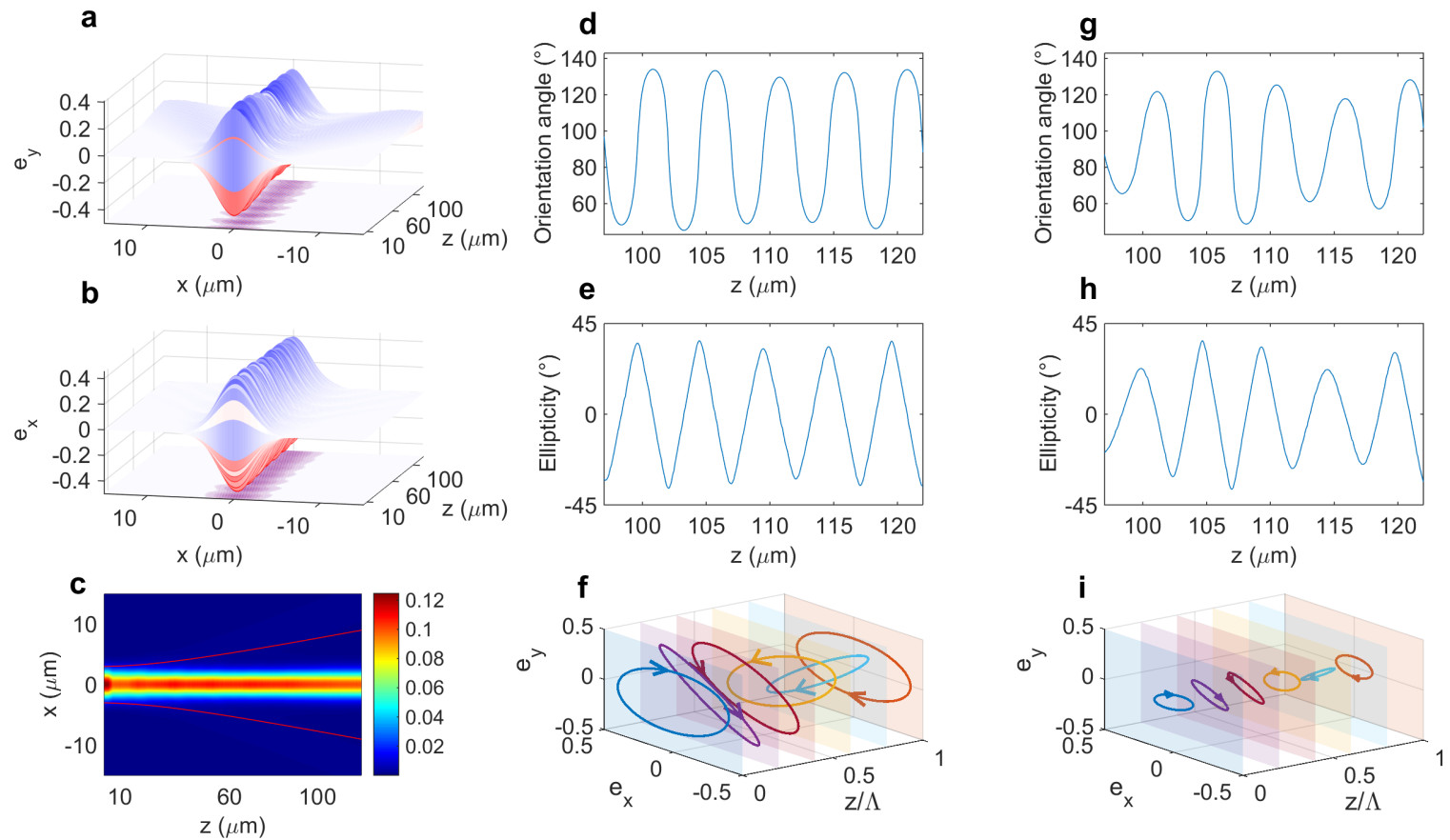

FIG. S2. Numerical snapshots of $(\mathbf{a}) e_{y}$ and (b) $e_{x}$ in the plane $x z$ once the stationary regime is reached in time; (c) corresponding average intensity map in $x z$; the red solid lines correspond to beam diffraction when $\Gamma_{0}=0$, i.e., the homogeneous case. (d, g) Angular orientation of the polarization ellipse and $\mathbf{e}, \mathbf{h}$ ) field ellipticity (arctangent of the ratio of minor to major axes) versus $z$ in (d-e) $x=0$ and (g-h) $x=3 \mu \mathrm{m}$, respectively. Polarization ellipses in (f) $x=0$ and (i) $x=3 \mu \mathrm{m}$ plotted versus $z$ in an interval $0 \leq z \Delta n / \lambda \leq 1$ far away from the excitation point, corresponding to $97 \mu \mathrm{m} \leq z \leq 102 \mu \mathrm{m}$. Here $w_{D}=5$ $\mu \mathrm{m}, \Gamma_{0}=15^{\circ}, \mathrm{RCP}$ input.
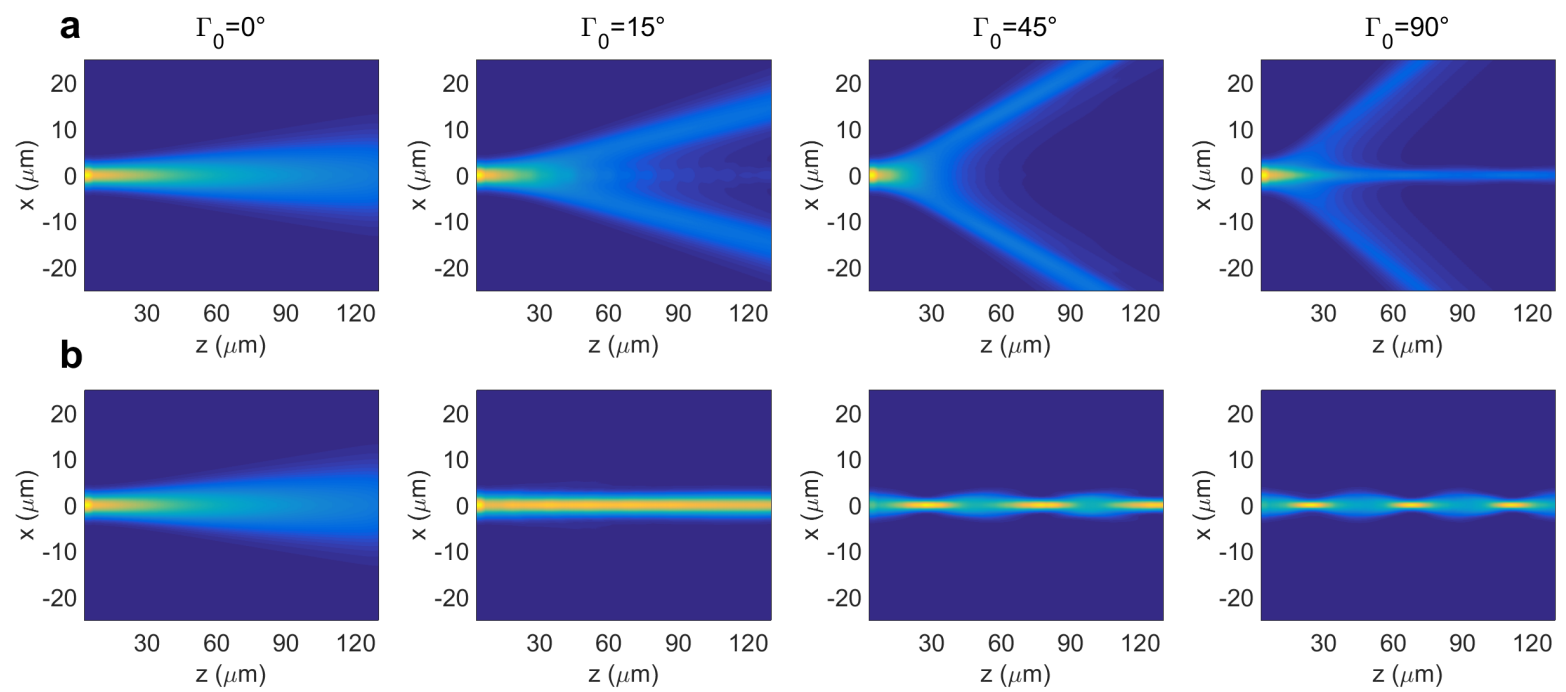

FIG. S3. Average beam intensity in the plane $x z$ when the input is (a) LCP or (b) RCP. The maximum rotation $\Gamma_{0}$ is 0,15 , 45 and $90^{\circ}$, from left to right, respectively. Here $z_{0}=0$ and $w_{D}=5 \mu \mathrm{m}$.

$\bar{n} /\left(2 Z_{0}\right)\left(\left|E_{x}\right|^{2}+\left|E_{y}\right|^{2}\right)$ with $Z_{0}$ the vacuum wave impedence, thus ignoring impedance variations for the two polarizations. 

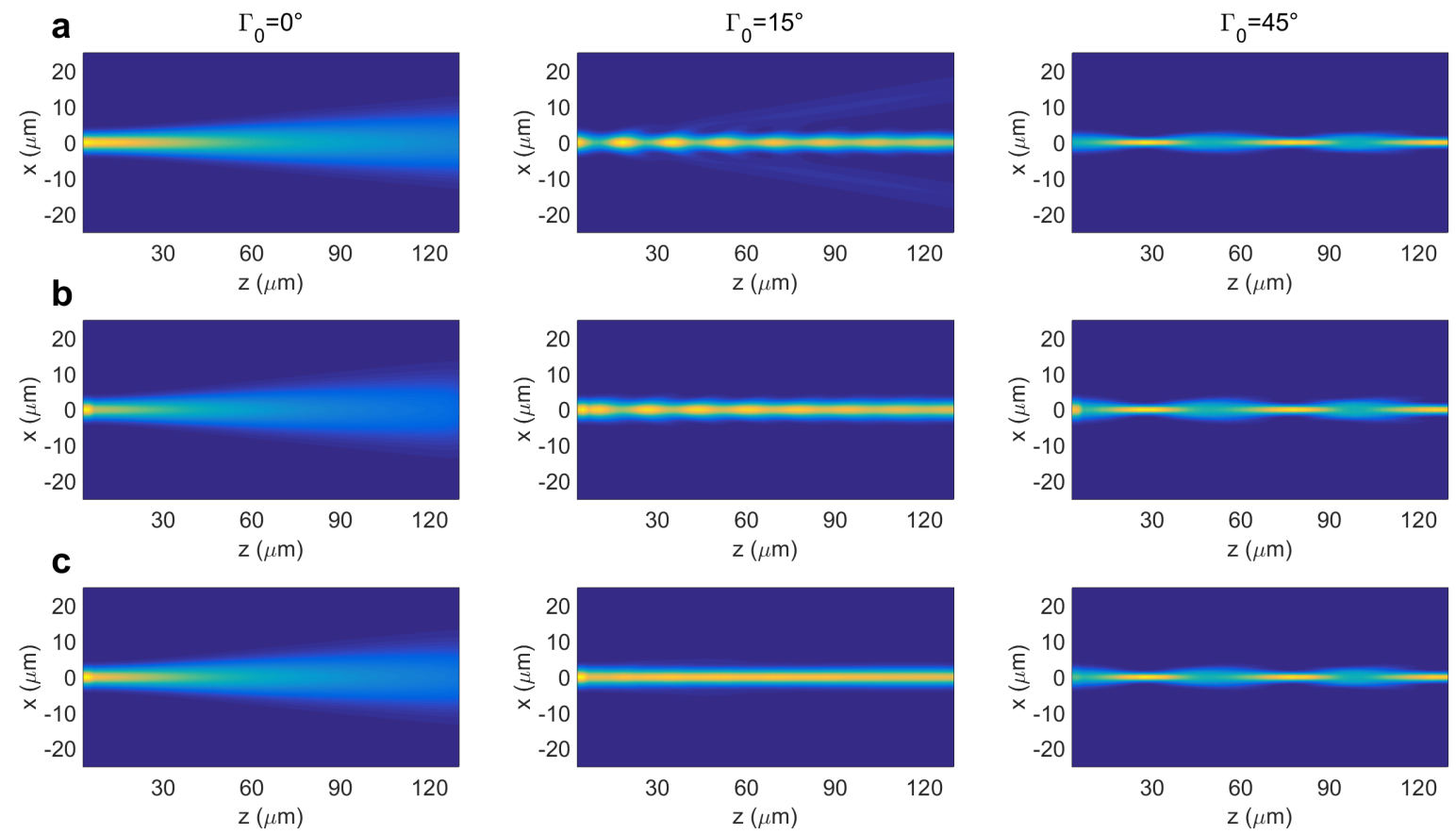

FIG. S4. Evolution maps of (a) $\left|E_{y}\right|^{2}$, (b) $\left|E_{x}\right|^{2}$ and (c) overall average intensity in the propagation plane $x z$. The maximum rotation $\Gamma_{0}$ is equal to 0,15 and $45^{\circ}$ from left to right, respectively. The input wave is RCP. Here $w_{D}=5 \mu \mathrm{m}$.

\section{Dependence on the transverse size of the effective waveguide}
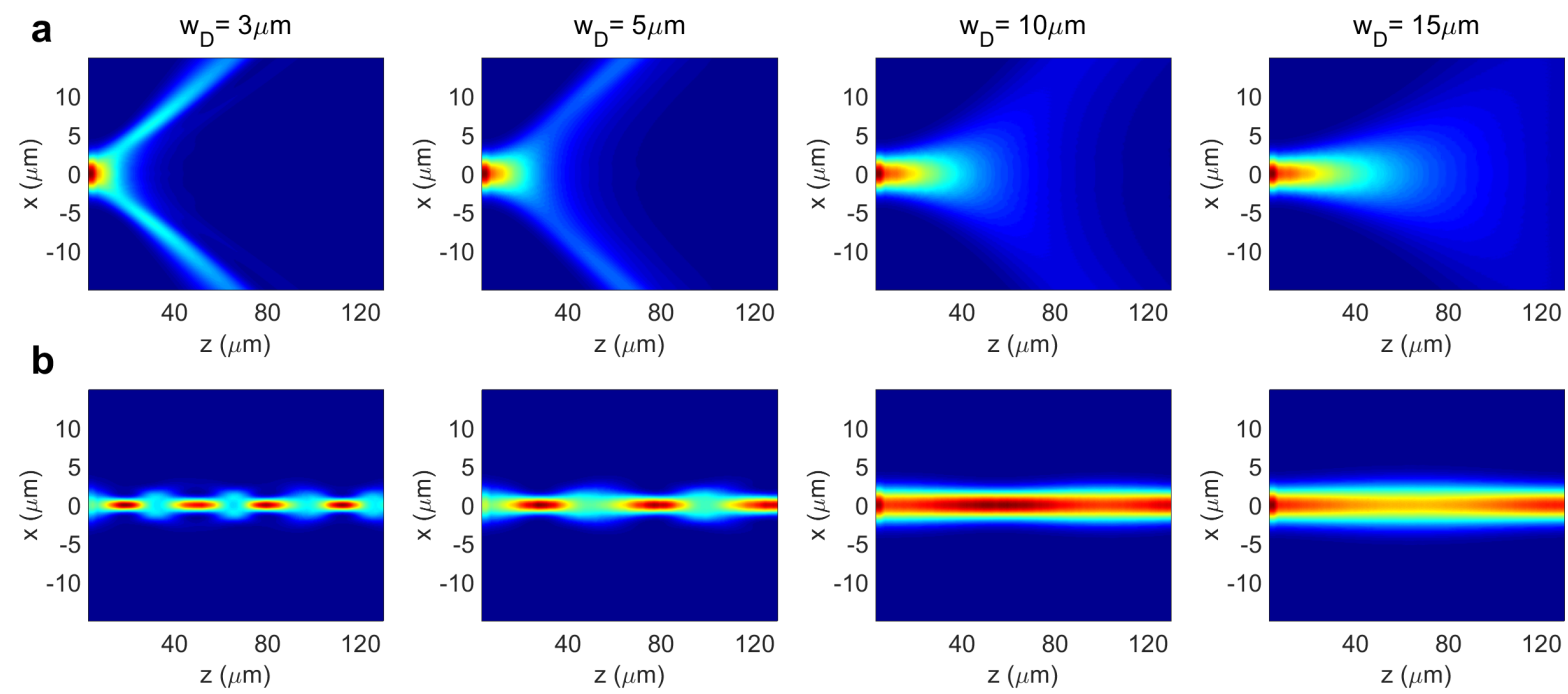

FIG. S5. Average intensity evolution in the propagation plane $x z$ for (a) left- and (b) right-handed circularly polarized wavepackets. Here $\Gamma_{0}=45^{\circ}$ whereas the angular distribution width $w_{D}$ is $3,5,10$ and $15 \mu \mathrm{m}$ from left to right, respectively.

Another important feature of the system is its dependence on the transverse extent of the orientation angle distribution. Both circular polarizations are shown in Supplementary Fig. S5. In agreement with theory, the smaller $w_{D}$ the stronger is the repulsion of the defocused component from the perturbed region. Light spatial localization increases as $w_{D}$ reduces, with intensity oscillations becoming slower in space as the $\theta$ distribution gets wider and wider. In line with Eqs. (S15, S16), light confinement undergoes the same trend as in standard waveguides based on total internal reflection. 


\section{E. Dependence on the input point}
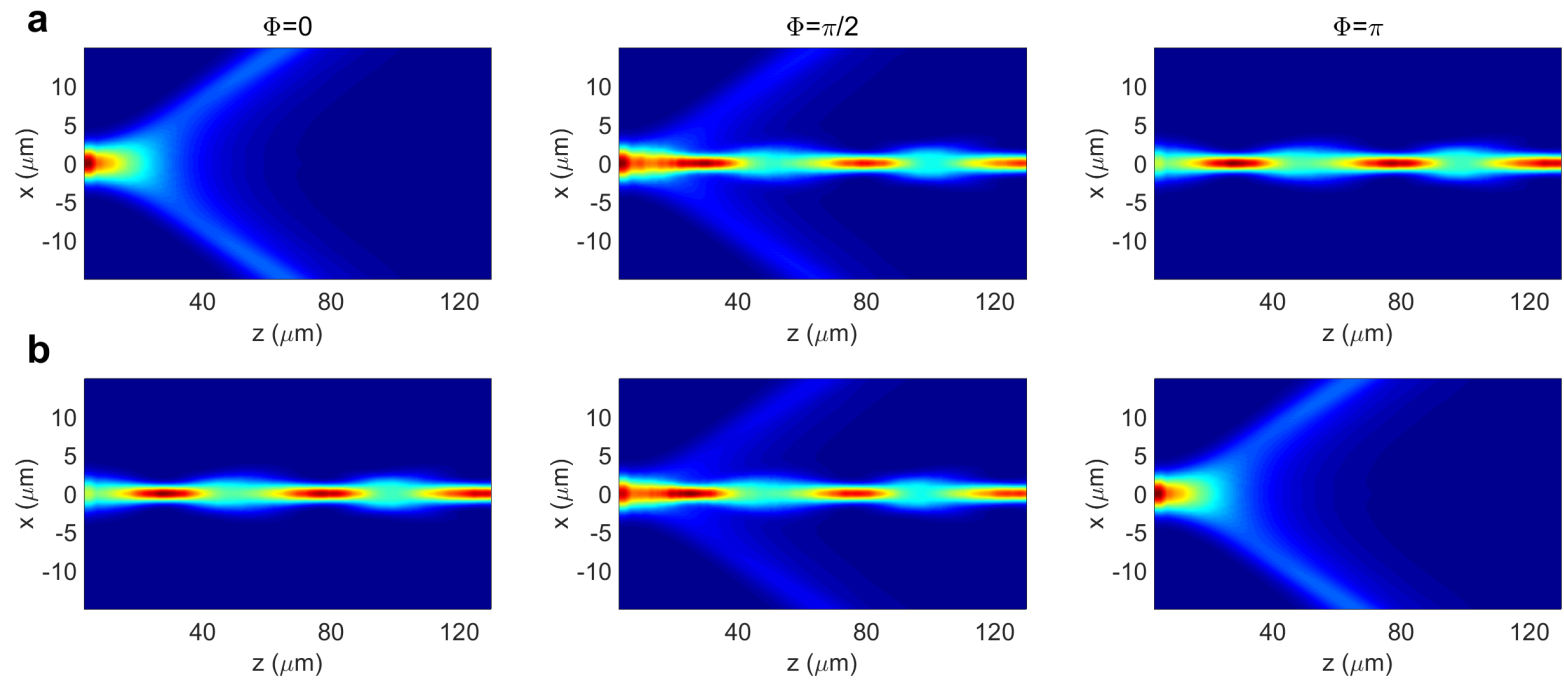

FIG. S6. Wavepacket evolution in $x z$ for input LCP (a) and RCP (b) when the longitudinal modulation shift $\Phi$ is $0, \pi / 2$ and $\pi$, from left to right, respectively. Here $\Gamma_{0}=45^{\circ}$ and $w_{D}=5 \mu \mathrm{m}$.

Finally, we studied light propagation as the longitudinal modulation $\sigma(z)$ was shifted, that is, as the phase $\Phi$ in $\sigma=\sin \left[\frac{2 \pi \Delta n}{\lambda}\left(z-z_{0}\right)+\Phi\right]$ was modified. Supplementary Fig. S6 shows the FDTD results: as predicted (Eqs. (S17 S18), when the phase is inverted (i.e., $\Phi=\pi$ ) the two circular polarizations exchange roles, with RCP waves going from trapping to anti-guiding and the opposite for LCP; when $\Phi=\pi / 2$, the intensity evolution remains the same regardless the input ellipticity: this agrees with Supplementary Fig. S2 showing quasi-linear polarization at a quarter period.

38 The propagation constant remains wavelength dependent through the vacuum wavevector on the LHS of Eqs. S15. S16

39 This contribution is $O\left(\Lambda^{2}\right)$ and was neglected when deriving Eqs. (S7) S8): in the complete expression the term $\sum_{p>1}\left(\int \frac{\partial \beta_{p}^{(j)}}{\partial x} d z \int \frac{\partial \beta_{-p}^{(j)}}{\partial x} d z\right)(j=e, o)$ appears within square brackets and brings in the role of all the fast scales on the slow scale. 